\title{
Addison disease
}

INSERM

\section{Source}

INSERM. (1999). Orphanet: an online rare disease and orphan drug data base. Addison disease. ORPHA:85138

Addison disease (AD) is a chronic and rare endocrine disorder due to autoimmune destruction of the adrenal cortex and resulting in a glucocorticoid and mineralocorticoid deficiency. Properly speaking AD designates autoimmune adrenalitis, but it is a term commonly used to describe any form of chronic primary adrenal insufficiency (CPAl; see this term). 